\title{
Nosocomial infection among patients with COVID-19: A retrospective data analysis of 918 cases from a single center in Wuhan, China
}

\author{
Yan He PhD ${ }^{1}$ (1), Wei Li MD¹, Zhen Wang MD¹, Huilong Chen $\mathrm{PhD}^{2}$, Lei Tian MD³ and Dong Liu PhD ${ }^{1}$ (1) \\ ${ }^{1}$ Department of Pharmacy, Tongji Hospital Affiliated to Tongji Medical College, Huazhong University of Science and Technology, Wuhan, China, ${ }^{2}$ Department of \\ Infectious Diseases, Tongji Hospital, Tongji Medical College, Huazhong University of Science and Technology, Wuhan, China and ${ }^{3}$ Department of Microbiological \\ Laboratory, Tongji Hospital, Tongji Medical College, Huazhong University of Science and Technology, Wuhan, China
}

To the Editor-The emergence of coronavirus disease-2019 (COVID-19) in China at the end of 2019 has caused a global pandemic and is a major public health issue. ${ }^{1}$ The percentage of nosocomial infection among COVID-19 patients who have died was significantly higher than that of patients who were cured and discharged $(P=.002){ }^{2}$ We investigated nosocomial infection among COVID-19 patients, and we analyzed risk factors to provide basic data for nosocomial infection prevention and control.

We retrospectively analyzed the clinical data of 918 COVID-19 patients in Tongji Hospital from December 30, 2019, to February 29, 2020. We performed a 1:4 paired case-control study: 65 patients with nosocomial infection were assigned to the case group and 260 non-nosocomial infection patients were assigned to the control group. We analyzed clinical data regarding patient demographics, basic disease, and treatments, and we summarized influencing factors of NI among COVID-19 patients. This study was approved by the Ethics Commission of Tongji Hospital (no. TJ-IRB20200338).

Male gender accounted for $47.7 \%$ of the 65 patients in case group (Table 1). The median age at the time of admission was 51 years (IQR, 36-71 years). In total, 40 of 65 patients $(65.6 \%)$ in the case group had comorbidities; the most prevalent of these were hypertension (36.9\%), cardiovascular disease (18.5\%), and diabetes (18.5\%). Approximately one-third of patients (33.8\%) had COVID-19 at the time of admission. ${ }^{3}$ The nosocomial infection rate among COVID-19 patients was 7.1\% (65 of 918). The most common nosocomial infection was pneumonia (32.3\%), followed by bacteremia (24.6\%), and urinary tract infection (21.5\%). In total, among the 43 pathogens isolated from nosocomial infections, 17 were gram-positive bacteria, 21 were gramnegative bacteria, and 5 were fungi. Onset of nosocomial infection occurred as early as day 7 of the course of illness and as late as day 22 , with an average of $14.3 \pm 8 \mathrm{~d}$. The mortality of COVID-19 patients with nosocomial infection was $15.4 \%$, significantly higher than that of COVID-19 patients without nosocomial infection (7.3\%; odds ratio [OR], 3.87; 95\% confidence interval $[\mathrm{CI}], 0.84-4.16 ; P=.045)$.

The association between demographic and clinical factors and the treatment of nosocomial infection as determined by univariate and multivariable analyses was displayed in Supplementary

Author for correspondence: Liu Dong, E-mail: ld2069@outlook.com; Yan He, E-mail: heyan_may@hotmail.com

Cite this article: $\mathrm{He} \mathrm{Y}$, et al. (2020). Nosocomial infection among patients with COVID-19: A retrospective data analysis of 918 cases from a single center in Wuhan, China. Infection Control \& Hospital Epidemiology, 41: 982-983, https://doi.org/10.1017/ ice. 2020.126
Table 1. Characteristics of Nosocomial Infection Among Patients With COVID-19

\begin{tabular}{|c|c|c|}
\hline Characteristic & No. $(\mathrm{N}=65)$ & $\%$ \\
\hline Age, median y (IQR) & $51(27-68)$ & \\
\hline \multicolumn{3}{|l|}{ Sex } \\
\hline Male & 31 & 47.7 \\
\hline Female & 34 & 52.3 \\
\hline Comorbidities & 40 & 65.6 \\
\hline Hypertension & 24 & 36.9 \\
\hline Cardiovascular disease & 12 & 18.5 \\
\hline Diabetes & 12 & 18.5 \\
\hline Underlying hematological disease & 3 & 4.6 \\
\hline Chronic kidney disease & 3 & 4.6 \\
\hline Respiratory disease & 2 & 3.1 \\
\hline Cerebrovascular disease & 2 & 3.1 \\
\hline Chronic liver disease & 1 & 1.5 \\
\hline Malignancy & 1 & 1.5 \\
\hline \multicolumn{3}{|l|}{ Charlson comorbidity score } \\
\hline 2 & 46 & 70.8 \\
\hline $3-4$ & 19 & 29.2 \\
\hline \multicolumn{3}{|l|}{ Clinical classification of COVID-19 } \\
\hline Severe type & 43 & 66.0 \\
\hline Critical type & 22 & 33.8 \\
\hline Invasive devices (CVC or PICC) & 25 & 38.5 \\
\hline Prophylactic application of antibiotics & 49 & 75.4 \\
\hline Cephalosporins & 6 & 9.2 \\
\hline Fluoroquinolones & 40 & 61.5 \\
\hline$\beta$ lactam $/ \beta$-lactamase inhibitors & 5 & 7.7 \\
\hline Azithromycin & 3 & 4.6 \\
\hline Ornidazole & 2 & 3.1 \\
\hline Combination of antibiotics $^{a}$ & 7 & 10.8 \\
\hline \multicolumn{3}{|l|}{ Infection site } \\
\hline Pneumonia & 21 & 32.3 \\
\hline Bacteremia & 16 & 24.6 \\
\hline
\end{tabular}

(C) 2020 by The Society for Healthcare Epidemiology of America. All rights reserved. This is an Open Access article, distributed under the terms of the Creative Commons Attribution licence (http://creativecommons.org/licenses/by/4.0/), which permits unrestricted re-use, distribution, and reproduction in any medium, provided the original work is properly cited. 
Table 1. (Continued)

\begin{tabular}{|c|c|c|}
\hline Characteristic & No. $(\mathrm{N}=65)$ & $\%$ \\
\hline Urinary tract infection & 14 & 21.5 \\
\hline Skin soft-tissue infection & 8 & 12.4 \\
\hline Gum infection & 4 & 6.2 \\
\hline Others & 2 & 3.1 \\
\hline Antiviral treatment & 46 & 70.8 \\
\hline Glucocorticoid treatment & 25 & 38.5 \\
\hline Pathogen isolates & 43 & \\
\hline Coagulase negative staphylococcus & 12 & 27.9 \\
\hline Acinetobacter & 9 & 20.9 \\
\hline Pseudomonas aeruginosa & 6 & 14.0 \\
\hline Enterococcus faecium & 5 & 11.6 \\
\hline Klebsiella pneumoniae & 4 & 9.3 \\
\hline Escherichia coli & 2 & 4.6 \\
\hline Candida albicans & 2 & 4.6 \\
\hline Mucor & 2 & 4.6 \\
\hline Other & 1 & 2.3 \\
\hline Mortality & 10 & 15.4 \\
\hline
\end{tabular}

Note. IQR, interquartile range.

Table 1. Significant positive associations between nosocomial infection and the following were detected by univariate analysis: diabetes, hematological disease, invasive devices (central venous catheter [CVC] or peripherally inserted central catheter [PICC]), combination of antibiotics, and glucocorticoid treatment. Among these factors, the highest odds ratio was for invasive devices (OR, 4.62; 95\% CI, 2.47-8.62) followed by diabetes (OR, 3.04; 95\% CI, 1.38-6.69), combination of antibiotics (OR, 3.02; 95\% CI, 1.10-8.26), glucocorticoid treatment (OR, 2.44; 95\% CI, 1.36-4.37), and hematological disease (OR, 1.95; 95\% CI, 1.01-1.06).

For multivariable analysis, the dependent variable was nosocomial infection status and independent variables were all factors that demonstrated statistical significance, as mentioned with univariate analysis. Significant predictors of nosocomial infection after adjustment for other covariates were invasive devices (OR, 4.28; 95\% CI, 2.47-8.61; $P=.007$ ) followed by diabetes (OR,: 3.06, 95\% CI, 1.41-7.22; $P=.037)$, and combination of antibiotics (OR, 1.84, 95\% CI, 1.31-4.59; $P=.003$ ) (Supplementary Table 1 online).

In conclusion, these findings suggest that nosocomial infections are common among patients with COVID-19 and can be predicted by considering certain risk factors. Rational utilization of antibiotics and steroids to treat patients with COVID-19 is important in preventing nosocomial infection, and special attention should be given to diabetic patients and patients with invasive devices (ie, CVC or PICC). Future studies are warranted to evaluate the efficacy of implementing infection control strategies or protocols on COVID-19 patients to achieve better therapeutic outcomes.

\section{Acknowledgments. None.}

Financial support. This work was financially supported by the National Natural Science Foundation of China (grant no. 81803387) and the Hubei Provincial Natural Science Foundation of China (grant no. 2018CFB152).

Conflicts of interest. All authors report no conflicts of interest relevant to this article.

\section{References}

1. Chen N, Zhou M, Dong X, et al. Epidemiological and clinical characteristics of 99 cases of 2019 novel coronavirus pneumonia in Wuhan, China: a descriptive study. Lancet 2020;395:507-513.

2. Ruan Q, Yang K, Wang W, Jiang L, Song J. Clinical predictors of mortality due to COVID-19 based on an analysis of data of 150 patients from Wuhan, China. Intensive Care Med 2020 [Epub ahead of print]. doi: 10.1007/s00134020-05991-x.

3. Diagnosis and treatment protocol for novel coronavirus pneumonia, trial version 7. World Health Organization website. https://www.who.int/docs/ default-source/wpro-documents/countries/china/covid-19-briefing-nhc/ 1-clinical-protocols-for-the-diagnosis-and-treatment-of-covid-19-v7.pdf? sfvrsn=c6cbfba4_2. Published March 3, 2020. Accessed April 15, 2020.

\title{
SARS-CoV-2: The Lombardy scenario in numbers
}

\author{
Andrea Molinari $M D^{1}$ (10), Federico Pistoia $M D^{1}$ and Giuditta Antonelli JD ${ }^{2}$ \\ ${ }^{1}$ Department of Health Sciences (DISSAL), University of Genova, Genoa, Italy and ${ }^{2}$ University of Genova, Genoa, Italy
}

To the Editor-On March 11, 2020, the World Health Organization (WHO) declared the spread of severe acute respiratory syndrome coronavirus 2 (SARS-CoV-2) to constitute a pandemic of COVID-19 infectious disease. ${ }^{1}$ On February 20, 2020, the first national cluster in Italy was identified in the Lombardy region

Author for correspondence: Andrea Molinari, Email: a.molinari@live.it

Cite this article: Molinari A, Pistoia F, and Antonelli G. (2020). SARS-CoV-2: The Lombardy scenario in numbers. Infection Control \& Hospital Epidemiology, 41: 983-984, https://doi.org/10.1017/ice.2020.115 after the diagnosis of SARS-CoV-2 in a 38-year-old man with a severe pneumonia and no relevant exposure history. ${ }^{2}$ To date, 74,386 SARS-CoV-2 laboratory-confirmed cases have been reported in Italy, with 32,346 cases in Lombardy alone, by far the most affected region. ${ }^{3}$

Given the extent of the phenomenon, we must urgently consider how the rapid spread of the infection can overload the National Health Service (SSN) and affect the mortality rate. The SSN is regarded as a high-level healthcare service, and it is regionally 